\title{
Discussion: Treatment of Breast Animation Deformity in Implant- Based Reconstruction with Selective Nerve Ablation
}

\author{
Michelle Lee ${ }^{1}$
}

Received: 8 July 2018/Accepted: 17 July 2018/Published online: 31 July 2018

(C) Springer Science+Business Media, LLC, part of Springer Nature and International Society of Aesthetic Plastic Surgery 2018

Level of Evidence $V$ This journal requires that authors assign a level of evidence to each article. For a full description of these Evidence-Based Medicine ratings, please refer to the Table of Contents or the online Instructions to Authors www.springer.com/00266.

Animation deformity is a known complication after subpectoral breast implant placement whether the implant is placed for aesthetic or reconstructive purposes. Spear et al. studied animation deformity in the aesthetic population with 195 primary breast augmentation patients with implants placed in a dual plane. The authors found $82 \%$ of the patients reported mild to no distortion and $7 \%$ reported severe distortion. Among the survey responders, only $1 \%$ of the patients stated that she would not recommend subpectoral positioning [1]. Unlike the aesthetic population, the reconstructive patient population is more sensitive to the negative impacts of animation deformity. In a study by Becker and Fregosi of 25 patients after mastectomy with implants placed in a subpectoral plane, almost all patients had animation deformity and $80 \%$ of the patients were bothered by the animation deformity and $45 \%$ were bothered by it to a significant degree [2].

There has been renewed interest in breast reconstruction to place the implant in a prepectoral plane due to improvements in mastectomy flap thickness, acellular dermal matrix placement and awareness of animation deformity of the implants. However, placing the implants in a prepectoral plane at the time of mastectomy or exchanging the implant pocket to a prepectoral plane is not

Michelle Lee

Michelle.Lee@dupagemd.com

1 Dupage Medical Group, 28381 Davis Parkway Suite 700, Warrenville, IL 60555, USA a viable option for all patients. In the subpectoral implant patient population, animation deformity remains problematic.

The authors in this article present a technique to secondarily correct animation deformity. Using a four-centimetre incision along the superolateral aspect of the pectoralis major muscle eight centimetres from the insertion of the muscle, the authors directly identify distal branches of the medial and lateral pectoral nerves. An arbitrary number of distal branches of the medial and lateral pectoral nerves were selected and denervated through bipolar cautery. Deactivation of the nerve branches was then confirmed with a nerve stimulator. Preservation of some of the lateral pectoral nerve branches prevented the pectoralis major muscle from complete denervation. In this report of a single patient, the authors corrected the animation deformity without long-term muscle atrophy and demonstrated an excellent two-year result.

Although the authors demonstrated a nice long-term result, more patients are needed to validate the effectiveness of this technique. It is difficult to predict the consistency of the technique given an arbitrary number of nerve branches were weakened. How many distal branches need to be weakened to correct the animation deformity without causing atrophy of the muscle? How much atrophy of the pectoralis muscle can be tolerated prior to compromising breast aesthetics. Further, a more systematic approach to the selection of nerves to ablate will make it easier to teach the technique. Lastly, the major difference between the authors' technique and Bernini et al.'s technique of selectively ablating the lower two-thirds of the pectoralis major muscle is timing [3]. Bernini performs the denervation at the time of mastectomy, and the authors perform it secondarily. One can argue that if prepectoral reconstruction is 
not possible, selective denervation of the pectoralis muscle should be performed at the time of mastectomy to avoid animation deformity.

\section{Compliance with Ethical Standards}

Conflict of interest The authors declare that they have no conflict of interest.

\section{References}

1. Spear S, Schwartz J, Dayan J, Clemens M (2009) Outcome assessment of breast distortion following submuscular breast augmentation. Aesthetic Plast Surg 33(1):44-48

2. Becker H, Fregosi N (2017) The impact of animation deformity on quality of life in post-mastectomy reconstruction patients. Aesthet Surg J 37(5):531-536

3. Bernini M, Casella D, Mariotti C (2017) Selective pectoralis major muscle denervation in breast reconstruction: a technical modification for more effective and cosmetic results. Gland Surg 6(6):745-750 